\title{
LPS-TLR4 pathway stimulating in acute-on-chronic liver failure by
}

\author{
promote NETs formation
}

Yang Liu, Shuo Chen, Jiazhong Wang, Shuo Yu, Xin Zhang, Yiming Li, and Gang Cao

Authors' affiliations:

Department of General Surgery, Xi'an Jiaotong University Second Affiliated Hospital, Xi'an, China;

Backgrounds: Intrahepatic infiltration of neutrophils is a character of acute-onchronic liver failure (ACLF) and neutrophil extracellular traps (NETs) are an important strategy for neutrophils to fix and kill invading microorganisms. Intestinal bacteria and gut-liver axis have been thought to play a key role in many liver diseases also including ACLF. However, whether NETs appear in ACLF and paly role in ACLF still unsure. Methods: WT, NE KO and TLR4 KO mice were used to build ACLF model, and the intestinal bacteria were eliminated at the same time and LPS was given. Then the formation of NETs and ACLF related markers were detected. Results: The serum MPO-DNA and LPS concentration was increased in ACLF patients and correlation was revealed between these two indexes. More intrahepatic NETs formed in ACLF mice by testing MPO-DNA, Cit H3 and NE. These markers decreased with gut detergent and restored of these markers with gut detergent plus LPS supplement. While NETs formation failed to change with gut microbiome or combine LPS supplement in TLR4 KO mice. As we tested ACLF related characters, liver injury, intrahepatic fat deposition, inflammation and fibrosis alleviated with depletion of NE. These related marks also attenuated with gut sterilization by antibiotics and recovered with combine treat with antibiotics plus LPS. But the liver injury, intrahepatic fat, fibro deposition and liver inflammation related markers did show a signification difference in TLR4 KO mice when it received same treatment. Conclusion: Intestinal-derived LPS promotes NETs formation in ACLF through TLR4 pathway and further accelerate ACLF process by NETs.

\section{Introduction}

Alcohol-related injuries are one of the most common causes of preventable illness in the world, and leading 3.3 million people death each year and accounting for $6 \%$ of all deaths worldwide ${ }^{1}$. Alcohol abuse can insult many target organs and cause multiple system damage. Particularly the liver and gastrointestinal tract are primarily involved in alcohol metabolism, and alcohol abuse causes severe tissue damage. Alcoholic liver disease (ALD) includes a variety of liver diseases such as fatty liver, alcoholic cirrhosis hepatitis (ASH), fibrosis, cirrhosis, and hepatocellular carcinoma. Among them, acute- 
on-chronic liver failure (ACLF) or sacute hepatitis (AH) is a type of acute on chronic liver disease, the most serious form of ALD, and is characterized with a highly mortality. According to report, up to $40 \%$ of patients with severe ACLF die within 6 months because there is no effective cure method other than the initiation of prednisolone ${ }^{2}$. Therefore, intensive understanding the causes of ACLF is very important for its prevention and treatment.

Intrahepatic infiltration of neutrophils is one of the characters in $\mathrm{ACLF}^{3}$. Neutrophils is one of the major effector cells of the innate immune system and have a variety of immune functions such as phagocytosis, degranulation, reactive oxygen species production, while these functions partly rely on or together with neutrophil extracelluar traps (NETs) formation and release ${ }^{4}$. Alcohol intake, on the other hand, could causes decreased intestinal motility, overgrowth of intestinal bacteria, and destruction of the intestinal barrier, which can result in the transfer of excess bacteria and their metabolites (like LPS) into the liver ${ }^{5,6}$. LPS is thought to have the effect of promoting neutrophils to formation of NET by binding to its receptor TLR4. It has not yet been confirmed whether NET is formed in the liver of ACLF and whether intestinal derived LPS contribute the intrahepatic formation of NETs during the condition of ACLF.

NETs is an extracellular network structure capable of fighting toxic factors and killing bacteria, and is mainly composed of extracellular chains of depolymerized DNA and neutrophil granule proteins. These proteins include histones, neutrophil elastase(NE), myeloperoxidase (MPO), cathepsin G, and other more than 30 enzymes and proteins ${ }^{7}$. Although NETs can kill bacteria, fungi, viruses, parasites and further prevent spread of them and fight against serious infections, the excessive formation of NETs could also lead to exacerbation of inflammation, the development of immune disorders, and damage adjacent cells ${ }^{8,9}$. Therefore, NET is considered to paly role in many diseases such as cardiovascular disease and systemic lupus erythematosus (SLE) ${ }^{10}$. In liver, recent years NETs was also involved in liver ischemia-reperfusion injury, non-alcoholic disease, chemical induced chronic liver fibrosis, hepatocellular carcinoma et. $\mathrm{al}^{11-13}$. However, whether NETs is also related to ACLF still unsure. Based on the above information, we proposed the following hypothesis that intestinal-derived LPS may promotes intrahepatic NET formation via its receptor TLR4 and exacerbates the process of ACLF.

\section{Method}

\section{ACLF Model establishment:}

The C57 mouse was purchased from the Experimental Animal Center of Xi'an Jiaotong University while NE KO and TLR4 KO mice (C57 background) were purchased from Jackson Laboratory. Mice were kept bred in an SPF animal room with a 12-hour light-dark cycle and not restricted to water and food. Male C57 mice aged 8 to 10 weeks were used for experiment. During the first 6 weeks, mice were injected intraperitoneally with $\mathrm{CCl} 4(0.2 \mathrm{ml} / \mathrm{kg})$ dissolved in olive oil (1:4) twice weekly. At week 7, gastric construction catheterization were done by isoflurane anesthetized according to the method described by Shinji Furuya and mice were 
subcutaneously injected with buprenophine $(0.24 \mathrm{mg} / \mathrm{Kg})$ every 12 hours for 72 hours after surgery. At the same time, stop the injection of CCl4 for 1 week. From the 8th week, $\mathrm{CCl} 4(0.1 \mathrm{ml} / \mathrm{kg})$ was injected intraperitoneally, and meanwhile a customized animal high-fat diet (Future Biotech, Beijing, China ) containing alcohol was injected through the gastric tube. High-fat diet formula refers to the formula originally described by Thompson. This wine contains corn oil (37\% of calories) as fat, protein (23\%), carbohydrates $(5 \%)$ and corn oil as fat (37\% of the total). Calories), protein (23\%), carbohydrate $(5 \%)$, etc ${ }^{14-17}$. Alcohol content in the starts at $16 \mathrm{~g} / \mathrm{kg} /$ day and increases by $1 \mathrm{~g} / \mathrm{kg}$ every 2 days until $24 \mathrm{~g} / \mathrm{kg} /$ day.

\section{Gut detergent and LPS supplement}

Replace mouse drinking water with an aqueous solution containing neomycin $(0.3 \mathrm{~g}$ / L), ampicillin $(0.3 \mathrm{~g} / \mathrm{L})$, metronidazole $(0.3 \mathrm{~g} / \mathrm{L})$, and vancomycin $(0.15 \mathrm{~g} / \mathrm{L}) 2$ weeks before animal model building. Daily increase antibiotics concentration to reached concentration of neomycin $(1 \mathrm{~g} / \mathrm{L})$, ampicillin $(1 \mathrm{~g} / \mathrm{L})$, metronidazole $(1 \mathrm{~g} /$ $\mathrm{L})$, and vancomycin $(0.5 \mathrm{~g} / \mathrm{L})$ and then keep this concentration in drinking water until sample harvest. Stool was collected and tested colony forming unit (CFU) with blood agar plated to confirm bacteria was removed. In combine LPS treatment group, LPS $(300 \mu \mathrm{g} / \mathrm{kg} / \mathrm{d})$ was daily subcutaneous injected.

\section{Immunofluorescence and immunohistochemistry}

Bake the slices in an oven at $60^{\circ} \mathrm{C}$ for 30 minutes, then dewax with xylene and sink the slice into degraded concentration of alcohol. Then place it in a hot antigen recovery solution for 10 minutes. After blocking the antigen with goat serum (DakoCytomation), the primary antibody (1: 100) was added overnight. After washing, the fluorescently labeled secondary antibody (1: 200) was added and washed again, added DAPI. Then, the antifluorescent quencher was dropped and the cover glass was attached. In immunohistochemical staining, the primary antibody was added to the sections after antigen blocking, then the secondary antibody was added and washed, DAB was added for color development, and then the sections were washed and hematoxylin was added. After cleaning, soak in 75\%, 85\%, 95\%, 100\% ethanol and soak in xylene. Finally, cover the film for observation.

\section{Oil red stain}

After dehydrating the tissue with $20 \%$ and $30 \%$ sucrose solutions, the sample is embedded with an OCT embedding and placed in a cryostat to precool. Cut the tissue into $10 \mu \mathrm{m}$ slices and place on top of the slices. After soaking in distilled water and $60 \%$ isopropanol, soak in oil red dye solution for 5 minutes, in $60 \%$ isopropanol, stain with hematoxylin, mount the slide and observe.

\section{Determination of serum LPS concentration}

LPS concentration was detected using a commercially available color development LPS detection kit using the manufacturer's protocol. Briefly, a 1:50 diluted serum sample $\left(50 \mu \mathrm{L}\right.$ total) is incubated with $50 \mu \mathrm{L}$ LAL at $37^{\circ} \mathrm{C}$ for 10 minutes, then 100 $\mu \mathrm{L}$ of chromogenic substrate solution is added 6 minutes later for $37^{\circ} \mathrm{C}$ in the incubation $100 \mu \mathrm{L}$. Acetic acid was measured at an absorbance of $410 \mathrm{~nm}$ and reported as LPS concentration IU / $\mathrm{ml}$.

\section{Real-time PCR}


Fresh tissue was lysed in Trizol, chromofal was added to separate the layers, centrifugation was performed, the supernatant was added to isopropanol, and centrifugation was performed to collect the precipitate. Then use the DNA-free kit DNAse to remove DANs according to the protocol. Take $1 \mu \mathrm{g}$ of RNA and apply iScript ${ }^{\mathrm{TM}}$ Reverse Transcription Supermix to prepare the cDNA. Prepare a $20 \mu \mathrm{L}$ reaction system using $2 \mu \mathrm{L}$ of cDNA, primers, iQ ${ }^{\mathrm{TM}}$ SYBR Green Supermix, and water. Comparison of expression target genes mRNA and GAPDH, and relative amounts of gene expression provided for use with the $\mathrm{Ct}$ method of $\Delta \Delta$. The average expression of the target gene in the control group was set to 1.

\section{ELISA}

Liver samples were weighed and homogenized on ice with cell extraction buffer (Wanleibio. Co., China) containing protease inhibitors. After vortexing the sample for 30 minutes, the sample was centrifuged at $5000 \mathrm{rpm}$ to collect the supernatant and the protein content was quantified by BCA. Assay based on bovine serum albumin. Samples were diluted with PBS to equalize protein concentrations. First, the 96-well plate was coated with a coating solution and the plate was washed with wash buffer. The wells were then blocked with blocking buffer and standard solution and sample $(100 \mu \mathrm{L})$ were added to the wells. After incubation, the wells were washed, the detection antibody solution was added, and then the streptavidin-horseradish peroxidase solution was added. Next, the TMB substrate solution was added, and the stop solution was added. Added after 30 minutes of incubation. The absorbance was read at $450 \mathrm{~nm}$. The concentration was determined based on the standard curve.

\section{Statistics}

The results were analyzed by Prism 8.0 software. Numerical data is shown as mean \pm standard deviation. Student's t-test was used to compare the differences between the groups. A P value below 0.05 were considered significantly different.

\section{Result:}

\section{Increased NET formation in mouse ACLF model}

Although NETs formed in human ACLF, whether it also formed in rodent still unsure. So, we built a mice ACLF model as described to figure it out the question. This model is considered to imitate a series of human characteristic changes of ACLF such as hepatocyte damage, neutrophil infiltration, intestinal bacteria and fungi intrahepatic translocation via portal vein, liver inflammation and fibrosis ${ }^{19}$. After treated mice with alcohol, the serum ALT concentration and liver TG content were significantly higher than that in the sham group. Large amount of intrahepatic red-stained areas was observed revealed by both Oil-red staining and Sirius red staining. We also seen many $\alpha$-SMA and collagen-1a1 positive stained areas in liver by immunohistochemistry staining. These results suggest that ACLF mice model was successfully made. Compare with sham group, the serum LPS concentration was much higher in ACLF mice, and serum MPO-DNA concentration is significate increased in ACLF mice than sham treatment mice. Intrahepatic fluorescence of $\mathrm{NE}$ and $\mathrm{CitH} 3$ which revealed by immunofluorescence staining was significantly higher in ACLF mice than that in sham 
mice. These results suggest that a large number of NETs formed in the liver after chronic alcoholic liver injury in both human and rodent. Now we doubt that does NETs relate to ACLF and why NETs formed in this condition.

\section{Gut microbiota and LPS are important factors for NET formation in condition of ACLF}

Although our previous experiments suggest that NETs present in the liver of ACLF, what is responsible for this phenomenon is still unsure? In order to answer this question, a combine model of gut detergent and ACLF was used. The model of gut detergent with cocktail antibiotic has been consider to eliminate gut microbiome effectively and safely ${ }^{20,21}$. As a result, the intrahepatic fluorescence of Cit $\mathrm{H} 3$ and NE was significantly decreased after gut sterilization than control in ACLF mice model. Serum MPO-DNA concentration was also lower in antibiotics using group than no antibiotics control group. These suggests that gut microbiota is a critical factor for NET formation in ACLF. However, this cocktail antibiotics recipe unselectively removed almost all gut microbiome, which substance is key factor? Lipoteichoic acid, the PAMP of G+ bacteria, is believed to be the reason for the protective effect of intestinal probiotics in various studies. While LPS, the PAMPs of G- bacteria, have been found to have the function which stimulate NETs formation in many different experiments. Therefore, we speculated that LPS probably is the key factor among bacteria and play a critical role in NET formation in the condition of ACLF. So we give mice with LPS after removal intestinal bacteria, and found that supplement of LPS increased intrahepatic fluorescence of Cit H3 and NE than non LPS supplement mice in the combine gut detergent plus ACLF mice model. Meanwhile, we also tested serum MPO-DNA concentration and result shown that its level increased in combine given LPS group than no LPS supplement mice in gut detergent plus ACLF mice.

\section{TLR4 during alcohol consumption promotes the formation of NET}

TLR4 is thought to be the binding receptor for LPS. Many studies suggested that LPS promotes NET formation via TLR4 and further induced tissue damage. Therefore, we speculate whether TLR4 is also involved in liver damage exacerbated by NET during ACLF. A TLR4 KO mice was used to build the ACLF animal model, and results shown that serum MPO-DNA level of TLR4 KO mice was significantly lower than that of WT mice after alcohol administration. At same time, the fluorescence of intrahepatic $\mathrm{NE}$ and CitH3 in TLR4 KO mice was also significantly lower than the fluorescence of $\mathrm{NE}$ and Cit $\mathrm{H} 3$ in WT mice. Then we combined given TLR4 KO mice with antibiotics or antibiotics plus LPS in the model of ACLF and what interesting is that TLR4 KO mice shown different pattern compare with WT mice. Our results shown that there wasn't significant changes of serum MPO-DNA in TLR4 mice either when they received antibiotics or antibiotics plus LPS. Intrahepatic NE and Cit H3 were also detected by immunofluorescent method and we found that, not like WT mice, there wasn't significant different between control, antibiotics and antibiotics plus LPS group in TLR4 KO mice ACLF model. These results suggest that the intestinal derived LPS stimulate NETs formation by TLR4 in ACLF.

NETs is essential for ACLF in mice 
Since we found that the numerous of NETs present in the ACLF both in human and mice, and NETs has considered to damage adjacent cells in variety of bacterial and sterile pathological diseases or conditions. Therefore, we speculated that NET may also play some role in the etiology of ACLF. The NE KO mice was used in the study. Neutrophil elastase (NE) could translocate to the nucleus during NETosis and cleaves histones, thereby contributing to chromatin depolymerization ${ }^{4}$. So neutrophil won't respond to microorganisms and releasing NETs when NE absent ${ }^{22}$. In the experiment we found that NE KO mice had significantly lower serum ALT concentrations than WT mice in the model of ACLF. Liver TG content was also significantly lower in NE KO mice than that in WT mice. By oil-red staining of mouse liver, it was observed that the liver of WT mice had more red-stained areas than NE KO mice. We also tested the mRNAs of lipogenesis-related genes and found that the mRNA expression of ACC1 and FASN in NE KO mice was significantly lower than expression of WT mice. As for inflammation-related genes, the intrahepatic TNF $\alpha, \mathrm{Ccl} 2$, and $\mathrm{Cxcl} 1$ mRNA expression levels in NE KO mice were significantly lower than the level of WT mice. We also tested several indicators associated with liver fibrosis. By Sirius-red staining and immunochemistry staining of aSMA and collagen 1a1, it shown that WT mice had more intrahepatic positive staining area which indicate more fibro formation than NE KO mice. Meanwhile, the expression of TIMP2 and Collagen1 mRNA which associated with liver fibrosis was also significantly reduced in NE KO mice than WT mice by qPCR detection.

\section{Eliminate intestinal bacteria decreased ACLF in mice}

As we have demonstrated that NET promotes ACLF, and gut derived LPS promote NETs formation, and gut-liver axis and intestinal microbiome has believed to be cause for many alcohol and non-alcohol induced liver insults. We suspect whether gut bacteria and LPS relate to ACLF? We built a combination of intestinal detergent and ACLF model, and results show that antibiotics significantly reduced serum ALT concentration in ACLF. TG levels in the liver were significantly reduced without intestinal bacteria and oil red staining shown significantly reduced the intrahepatic redstained areas in antibiotics group than that in control group. The mRNA expression of ACC1 and FASN which is associated with liver tissue lipogenesis was also significate attenuated with the removal of gut microbiota. Expression of liver TNF $\alpha$, CCL2, Cxcl1 mRNA which are associated with inflammation were lower when mice received antibiotics compared with control. Intrahepatic fibro deposition was also reduced with gut sterlization by detection with Sirius-red staining and staining with $\alpha$ SMA and collagen 1a1. The expression of liver TIMP2 and collagen 1 mRNA decreased with gut detergent compare with control group.

\section{LPS is the key for intestinal bacteria insult liver in ACLF}

Next, we investigated whether LPS is the key for promoting effect of intestinal microbiome in ACLF. When LPS was co-administered to antibiotics treated mice, serum ALT concentration and liver TG content were significantly restored compared to antibiotics treated ACLF mice. Intrahepatic fatty deposition was revealed by oil red staining and result shown that more red staining area was seen after LPS supplement than no LPS supplement in gut sterilized ACLF mice. Intrahepatic fiber which detected 
by Siruis-red staining and immunochemistry staining of $\alpha$ SMA and collagen 1a1 was re-increased after given LPS to gut detergent mice. Liver lipogenesis related gene ACC1 and FASN, inflammation related gene TNF $\alpha$, CCL2, Cxc11, fibrosis related genes TIMP2 and Collagen 1 also had a higher expression level in LPS plus antibiotics treatment group than only antibiotics treatment group.

\section{ACLF Attenuate when mice absent TLR4}

Regard to TLR4 played a key role in intestine LPS induce NETs formation, it probably also related to ACLF process. In the study, we found that TLR4 KO mice significantly decreased serum ALT concentration than WT mice. The red-stained areas of the TLR4 KO mice liver were significantly less than WT mice by red oil-red staining, and the liver TG content was also significantly lower in TLR4 KO mice than WT mice. Expression of ACC1, FASN TNF $\alpha$, CCL2 and Cxcl1 mRNA in the liver of TLR4 KO mice was significantly decreased than WT mice. Sirius staining and immunohistochemical staining of $\alpha$-SMA and collagen 1a1 revealed that TLR4 KO mice had less positively stained region than WT mice. At the same time, the liver mRNA expression levels of TIMP2 and collagen1 were significantly alleviated in TLR4 KO mice than those of WT mice.

\section{TLR4 deficient mice fail to response intestinal microbiome and LPS in ACLF}

Interestingly, unlike WT mice in the model of ACLF, TLR4 KO mice did not show significant diversity when they removed intestinal microbiome or supplement LPS after gut sterilized. We tested serum ALT concentration and liver TG content, and results shown that there wasn't significate difference between control group, antibiotics group and antibiotics plus LPS group in TLR4 KO mice of ACLF model. Intrahepatic red staining areas and ACC1, FASN mRNA expression were same among these three groups in TLR4 KO mice ACLF model. Fibrous deposits also didn't show different between control, antibiotics and antibiotics plus LPS group in TLR4 KO mice ACLF model by detecting with Sirius red staining, immunohistochemical staining. Liver fibrosis related genes TIMP2, Collagen1 together with inflammation related genes TNF $\alpha$, CCL2, Cxcl1 mRNA expression kept in same level when removed gut bacteria and removed gut bacteria plus LPS in TLR4 KO mice.

\section{Discussion}

Human ACLF is main charactered with systemic inflammation, hepatocellular injury, intrahepatic infiltration of neutrophils, and surrounding liver fibrosis. However, most existing animal models of alcoholic liver disease are characterized by minor changes in liver histology, lack of neutrophil infiltration into the liver, and severe pericellular fibrosis ${ }^{23}$, this limited the study in the field of alcoholic liver disease, especially the ACLF. In this study, we used a model which introduced by Shinji Furuya who succeeded in reproducing the characteristics of human ACLF such as more severe liver damage, steatohepatitis, neutrophil infiltration into the liver, liver fibrosis, over growth of Escherichia coli and Candida ${ }^{24}$. So, this model is considered as an ideal model for ACLF research ${ }^{19}$.

It has been found that neutrophils increased in peripheral blood and liver tissue tropism of ALD patients and a higher intrahepatic neutrophil level which revealed by liver biopsies indicate a poor clinical outcome in ACLF patients ${ }^{25}$. Kupffer cells which 
activated after alcohol intake together with damaged liver cells recruit neutrophils to the liver by releasing chemical factors and cytokines. Although their specific mechanism of action is still not fully clear, these infiltrating neutrophils (including immature neutrophils) could lead liver damage in various ways ${ }^{26}$.

Neutrophil is the main source of ROS. Although ROS formation is an effective sterilization process, excessive or uncontrolled ROS formation can lead to unwanted tissue damage. It had showed that peripheral neutrophils isolated from ACLF or alcohol-related cirrhosis patients had a much higher ROS level compared to neutrophils from healthy donors, which result indicate that more neutrophils were activated in ALD patients $^{25}$. It's found that serum concentration of some NETs related proteins like lactoferrin, NE, lipoprotein 2 (LCN2) increased in ALD patients which imply more neutrophils may formed NETs in this condition ${ }^{27}$. Recently, NET has been also found to induce intrahepatic inflammation in an short term alcohol intake induced liver injury model $^{28}$, whether NET present in human and mouse ACLF is still unknown. In the study we found for the first time that MPO-DNA was elevated in human ACLF through clinical sampling, which suggesting the present of NET in human body of ACLF patients. However, our result was based on the test of blood sample and could not determine whether NET located in the liver or some other extrahepatic organs, so we further built an ACLF model in mice and found that a large amount of NETs present in the liver by liver sampling. Then we thought two questions, one is why NETs formed in liver in this condition, another is that is there some relationship between NETs and ACLF. In order to figure out these questions, a series of research were conducted.

It's well known that alcohol could affects multiple end organs (mainly the liver, intestines, and brain). In gut, it's found that alcohol could cause overgrowth of small intestinal bacteria after ingestion. It's found that ALD patients had a lower levels of Bifidobacterium, Lactobacillus spp., Faecalibacterium prausnitzii, Ruminoccoccus spp. as well as Bacteroidaceae, while the proportion of some G- bacteria like Lachnospiraceae was found increased ${ }^{17,29}$. On the other hand, alcohol could damage the intestinal barrier function and induce intestine hyperpermeability ${ }^{30}$. Although intestinal lumen content of IgA level increased in alcoholics, alcohol consumption impacts several key components of the non-immunologic intestinal barrier and associated with a decrease of many intestinal tight junction proteins ${ }^{30}$. As a result, excessive intestinal bacteria and it's component like LPS migrated into the liver via the portal vein which results was also confirmed in our experiment. In this study, less NET formation was observed with remove of intestinal bacteria in the process of ACLF. This result suggests that certain microbes or their components may be involved in the formation of NET in this condition. Many in vivo and in vitro studies had proved that LPS, the main component of G- bacterial cell membranes, has been identified as a strong stimulate affect on NETosis ${ }^{31-34}$. While little study shown that lipoteichoic acid (PAMPs of G+ bacteria) or bacterial DNA had a stimulating effect on NETosis and LTA even shown inhibitory effect on NETosis in some studies ${ }^{35}$.

When LPS translocate into liver, it can cause liver damage by various intrahepatic cells through combine with its receptor TLR4. It's reported that LPS activates Kuffer cells by interacting with CD14 and TLR4 on the surface of the Kuffer cells, then it will 
release ROS and various inflammatory cytokines and cause damage ${ }^{36}$. LPS could also activate sinusoidal endothelial cells via TLR4 and lead to release IL6 and result in liver damage. So, LPS and TLR4 had considered to has function to stimulate or regulate NETs formation in many bacterial or non-bacterial related disease or condition ${ }^{37-39}$. In another double insults model with alcohol and LPS, LPS could also activates hepatic stellate cells and increased deposits collagen fiber in the liver ${ }^{36}$. However, it is still unclear whether LPS-TLR4 can also insult liver by stimulation of neutrophil to form NET in the ACLF liver. In the study, we found that LPS restored the NETs formation in gut sterilized ACLF mice, and NETs formation didn't response with gut detergent or LPS in TLR4 KO mice. These results suggest that intestinal LPS could stimulate neutrophil to form NETs by TLR4 in ACLF.

Although NET play a crucial role in innate immunity, excessive NET formation is characterized by promoting inflammation and damaging host cells. Due to these effect, NETs is considered as underlying basis in many disease such as systemic lupus erythematosus (SLE), vasculitis, diabetes, thrombosis, lung damage ${ }^{40}$. In liver, NET has been shown to be involved in liver ischemia-reperfusion injury, NASH, hepatocellular carcinoma, etc ${ }^{11,12}$. More recently, Szabo's team proved that and NET has also involved in mice liver damage due to short-term acute alcohol intake ${ }^{28}$. In human, NETs present in ACLF and a higher intrahepatic neutrophil imply a poor outcome of ACLF. In this study, we found that liver damage, intrahepatic inflammation, fat deposition, fibrosis attenuates in the modle of ACLF without NETs which indicate that NETs take part in the process of ACLF. As gut-liver axis has been considered as the key factor for liver injury in virous of liver disease, also include ALD, and we proved that NETs paly role in ACLF and gut derived LPS stimulate NETs formation via its receptor TLR4, so it makes sense that intestinal LPS aggravate ACLF in the study. Although some study shown that alcohol impaired NET formation in response to antigen ${ }^{28,41}$, this probably due to in the setting of high circulating lipopolysaccharide (LPS) levels which prime neutrophils and impair their reactivity to further stimuli ${ }^{13}$. However, this study implies that LPS is essential for ACLF present in alcohol consumption at least.

In summary, we can conclude form study that human and mouse ACLF had a increased formation of NETs in the liver. Intestinal LPS causes excessive formation of NET in the liver via TLR4, which exacerbates the development of ACLF.

\section{Figure legend:}

Figure 1. Increased NETs marker and LPS in mice ACLF model. (A) Correlation between serum MPO-DNA and LPS concentration in ACLF patients. (B, C)Cit $\mathrm{H} 3$ and NE was detected by immunofluorescence method with liver sample. (D)Serum LPS concentration from sham and ACLF mice detected by ELISA method. *: $P<0.05$.

Figure 2. Gut derived LPS promote NETosis by TLR4 in the condition of ACLF. ACLF model was built in WT mice and the mice received gut detergent by cocktail antibiotics or antibiotics plus LPS supplement. Mice were sacrificed after 9 weeks of treatment and liver/serum samples analyzed by immunofluorescence for Cit H3 and NE (A, B), ELISA for MPO-DNA(C). ACLF mice 
model was also built with TLR4 mice and mice received gut detergent by cocktail antibiotics or antibiotics plus LPS supplement. After 9 weeks treatment, mice was euthanized for sample harvest. Cit $\mathrm{H} 3$ and NE was detected by immunofluorescence method with liver sample (D, E) and serum MPO-DNA content was revealed by ELISA method. *: $P<0.05$.

Figure 3. Depletion of NETs decreased liver injury, liver inflammation, fat deposiation and liver fibrosis in mice ACLF model. ACLF model was built in both WT and NE KO mice. Mice was euthanized at end of model building and liver or serum samples were collected. Serum ALT concentration was revealed by ELISA method (A). Liver fat deposition was detected by Oil red staining $(\times 200)$ and TG concentration detection $(B, C)$. Liver mRNAs of lipogenesis-related genes (ACC1, FASN) expression was detected by qPCR (D, E). Intrahepatic inflammation was revealed by TNF $\alpha$, CCL2, Cxcl1 with qPCR method (F-H). Mice were tested for fibrillar collagen by Sirius red staining $(\times 200)$ and expression of a-SMA and collagen 1a1 was determined by immunohistochemistry $(\times 400)(\mathrm{I}, \mathrm{J})$. Liver levels of fibrosis related genes (ACTA-2, TIMP-2 and Collagen-1) were determined by qPCR(J, K).

Figure 4. Mice ACLF process decreased without intestinal bacterial and this process recovered after LPS supplement. Intestinal microbiome was sterilized by cocktail antibiotics in ACLF mice model, and combine given mice with antibiotics plus LPS in another group of mice. Liver and serum samples were detected after sample collection at end of ACLF model building. Serum ALT concentration was detected by ELISA method. Intrahepatic fat deposition was revealed by Oil red staining $(\times 200)$ and liver TG content examination. Liver mRNAs of lipogenesis-related genes (ACC1, FASN) expression was detected by qPCR (D, E). Intrahepatic inflammation was revealed by TNF $\alpha$, CCL2, Cxcl1 with qPCR method (F-H). Mice were tested for fibrillar collagen by Sirius red staining $(\times 200)$ and expression of a-SMA and collagen 1a1 was determined by immunohistochemistry $(\times 400)(\mathrm{I}, \mathrm{J})$. Liver levels of fibrosis related genes (TIMP-2 and Collagen-1) were determined by qPCR(J, K).

Figure 5. TLR4 KO mice didn't response with antibiotics and LPS in ACLF model. ACLF model was built in TLR4 KO mice, and mice received different treatment with antibiotics or antibotics plus LPS. Liver and serum samples were collected at end of ACLF model building. Serum ALT concentration was detected by ELISA method. Intrahepatic fat deposition was revealed by Oil red staining $(\times 200)$ and liver TG content examination. Liver mRNAs of lipogenesis-related genes (ACC1, FASN) expression was detected by qPCR (D, E). Intrahepatic inflammation was revealed by TNF $\alpha$, CCL2, Cxcl1 with qPCR method $(\mathrm{F}-\mathrm{H})$. Mice were tested for fibrillar collagen by Sirius red staining $(\times 200)$ and expression of a-SMA and collagen 1a1 was determined by immunohistochemistry $(\times 400)(I, J)$. Liver levels of fibrosis related genes (TIMP-2 and Collagen-1) were determined by qPCR $(\mathrm{J}, \mathrm{K})$.

\section{References:}

1. Organization WH. Global status report on alcohol and health 2018: World Health 
Organization, 2018.

2. Altamirano J, Fagundes C, Dominguez M, et al. Acute kidney injury is an early predictor of mortality for patients with alcoholic hepatitis. Clin Gastroenterol Hepatol 2012;10:65-71 e3.

3. Mandrekar P, Bataller R, Tsukamoto H, et al. Alcoholic hepatitis: Translational approaches to develop targeted therapies. Hepatology 2016;64:1343-55.

4. Papayannopoulos V. Neutrophil extracellular traps in immunity and disease. Nat Rev Immunol 2018;18:134-147.

5. Bajaj JS. Alcohol, liver disease and the gut microbiota. Nat Rev Gastroenterol Hepatol 2019;16:235-246.

6. Posteraro B, Paroni Sterbini F, Petito V, et al. Liver Injury, Endotoxemia, and Their Relationship to Intestinal Microbiota Composition in Alcohol-Preferring Rats. Alcohol Clin Exp Res 2018;42:2313-2325.

7. Dabrowska D, Jablonska E, Garley M, et al. New Aspects of the Biology of Neutrophil Extracellular Traps. Scand J Immunol 2016;84:317-322.

8. Lee KH, Kronbichler A, Park DD, et al. Neutrophil extracellular traps (NETs) in autoimmune diseases: A comprehensive review. Autoimmun Rev 2017;16:1160-1173.

9. Jorch SK, Kubes P. An emerging role for neutrophil extracellular traps in noninfectious disease. Nat Med 2017;23:279-287.

10. Jeremic I, Djuric O, Nikolic M, et al. Neutrophil extracellular traps-associated markers are elevated in patients with systemic lupus erythematosus. Rheumatol Int 2019;39:18491857.

11. Liu $Y$, Qin $X$, Lei $Z$, et al. Tetramethylpyrazine inhibits neutrophil extracellular traps formation and alleviates hepatic ischemia/reperfusion injury in rat liver transplantation. Exp Cell Res 2021;406:112719.

12. van der Windt DJ, Sud $\mathrm{V}$, Zhang $\mathrm{H}$, et al. Neutrophil extracellular traps promote inflammation and development of hepatocellular carcinoma in nonalcoholic steatohepatitis. Hepatology 2018;68:1347-1360.

13. Hilscher MB, Shah VH. Neutrophil Extracellular Traps and Liver Disease. Semin Liver Dis 2020;40:171-179.

14. Thompson JA, Reitz RC. Effects of ethanol ingestion and dietary fat levels on mitochondrial lipids in male and female rats. Lipids 1978;13:540-50.

15. Morimoto M, Zern MA, Hagbjork AL, et al. Fish oil, alcohol, and liver pathology: role of cytochrome P450 2E1. Proc Soc Exp Biol Med 1994;207:197-205.

16. Kono H, Bradford BU, Rusyn I, et al. Development of an intragastric enteral model in the mouse: studies of alcohol-induced liver disease using knockout technology. J Hepatobiliary Pancreat Surg 2000;7:395-400.

17. Furuya S, Argemi J, Uehara T, et al. A Novel Mouse Model of Acute-on-Chronic Cholestatic Alcoholic Liver Disease: A Systems Biology Comparison With Human Alcoholic Hepatitis. Alcohol Clin Exp Res 2020;44:87-101.

18. Kessenbrock K, Krumbholz M, Schonermarck U, et al. Netting neutrophils in autoimmune small-vessel vasculitis. Nat Med 2009;15:623-5.

19. Furuya S, Cichocki JA, Konganti K, et al. Histopathological and Molecular Signatures of a Mouse Model of Acute-on-Chronic Alcoholic Liver Injury Demonstrate Concordance With 
Human Alcoholic Hepatitis. Toxicol Sci 2019;170:427-437.

20. Deshmukh HS, Liu Y, Menkiti OR, et al. The microbiota regulates neutrophil homeostasis and host resistance to Escherichia coli K1 sepsis in neonatal mice. Nat Med 2014;20:52430.

21. Liu M, Yuan J, Hu WJ, et al. Pretreatment with broad-spectrum antibiotics alters the pharmacokinetics of major constituents of Shaoyao-Gancao decoction in rats after oral administration. Acta Pharmacol Sin 2019;40:288-296.

22. Martinod K, Witsch T, Farley K, et al. Neutrophil elastase-deficient mice form neutrophil extracellular traps in an experimental model of deep vein thrombosis. J Thromb Haemost 2016;14:551-8.

23. Bertola A, Mathews S, Ki SH, et al. Mouse model of chronic and binge ethanol feeding (the NIAAA model). Nat Protoc 2013;8:627-37.

24. Ueno A, Lazaro R, Wang PY, et al. Mouse intragastric infusion (iG) model. Nat Protoc 2012;7:771-81.

25. Das S, Maras JS, Hussain MS, et al. Hyperoxidized albumin modulates neutrophils to induce oxidative stress and inflammation in severe alcoholic hepatitis. Hepatology 2017;65:631-646.

26. Honda M, Kubes P. Neutrophils and neutrophil extracellular traps in the liver and gastrointestinal system. Nat Rev Gastroenterol Hepatol 2018;15:206-221.

27. Tranah TH, Vijay GKM, Ryan JM, et al. Dysfunctional neutrophil effector organelle mobilization and microbicidal protein release in alcohol-related cirrhosis. Am J Physiol Gastrointest Liver Physiol 2017;313:G203-G211.

28. Bukong TN, Cho Y, Iracheta-Vellve A, et al. Abnormal neutrophil traps and impaired efferocytosis contribute to liver injury and sepsis severity after binge alcohol use. J Hepatol 2018;69:1145-1154.

29. Hartmann P, Schnabl B. New Developments in Microbiome in Alcohol-Associated and Nonalcoholic Fatty Liver Disease. Semin Liver Dis 2021;41:87-102.

30. Patel S, Behara R, Swanson GR, et al. Alcohol and the Intestine. Biomolecules 2015;5:257388.

31. Arroyo R, Khan MA, Echaide M, et al. SP-D attenuates LPS-induced formation of human neutrophil extracellular traps (NETs), protecting pulmonary surfactant inactivation by NETs. Commun Biol 2019;2:470.

32. Petretto A, Bruschi M, Pratesi F, et al. Neutrophil extracellular traps (NET) induced by different stimuli: A comparative proteomic analysis. PLoS One 2019;14:e0218946.

33. Sakurai K, Miyashita T, Okazaki M, et al. Role for Neutrophil Extracellular Traps (NETs) and Platelet Aggregation in Early Sepsis-induced Hepatic Dysfunction. In Vivo 2017;31:10511058.

34. Li RHL, Ng G, Tablin F. Lipopolysaccharide-induced neutrophil extracellular trap formation in canine neutrophils is dependent on histone $\mathrm{H} 3$ citrullination by peptidylarginine deiminase. Vet Immunol Immunopathol 2017;193-194:29-37.

35. Wartha F, Beiter K, Albiger B, et al. Capsule and D-alanylated lipoteichoic acids protect Streptococcus pneumoniae against neutrophil extracellular traps. Cell Microbiol 2007;9:1162-71.

36. Schaffert CS, Duryee MJ, Hunter CD, et al. Alcohol metabolites and lipopolysaccharide: 
roles in the development and/or progression of alcoholic liver disease. World J Gastroenterol 2009;15:1209-18.

37. Dong $Y$, Jin C, Ding Z, et al. TLR4 regulates ROS and autophagy to control neutrophil extracellular traps formation against Streptococcus pneumoniae in acute otitis media. Pediatr Res 2021;89:785-794.

38. Clark SR, Ma AC, Tavener SA, et al. Platelet TLR4 activates neutrophil extracellular traps to ensnare bacteria in septic blood. Nat Med 2007;13:463-9.

39. Shinde-Jadhav S, Mansure JJ, Rayes RF, et al. Role of neutrophil extracellular traps in radiation resistance of invasive bladder cancer. Nat Commun 2021;12:2776.

40. Mutua V, Gershwin LJ. A Review of Neutrophil Extracellular Traps (NETs) in Disease: Potential Anti-NETs Therapeutics. Clin Rev Allergy Immunol 2021;61:194-211.

41. Jin L, Batra S, Jeyaseelan S. Diminished neutrophil extracellular trap (NET) formation is a novel innate immune deficiency induced by acute ethanol exposure in polymicrobial sepsis, which can be rescued by CXCL1. PLoS Pathog 2017;13:e1006637. 
Figure 1
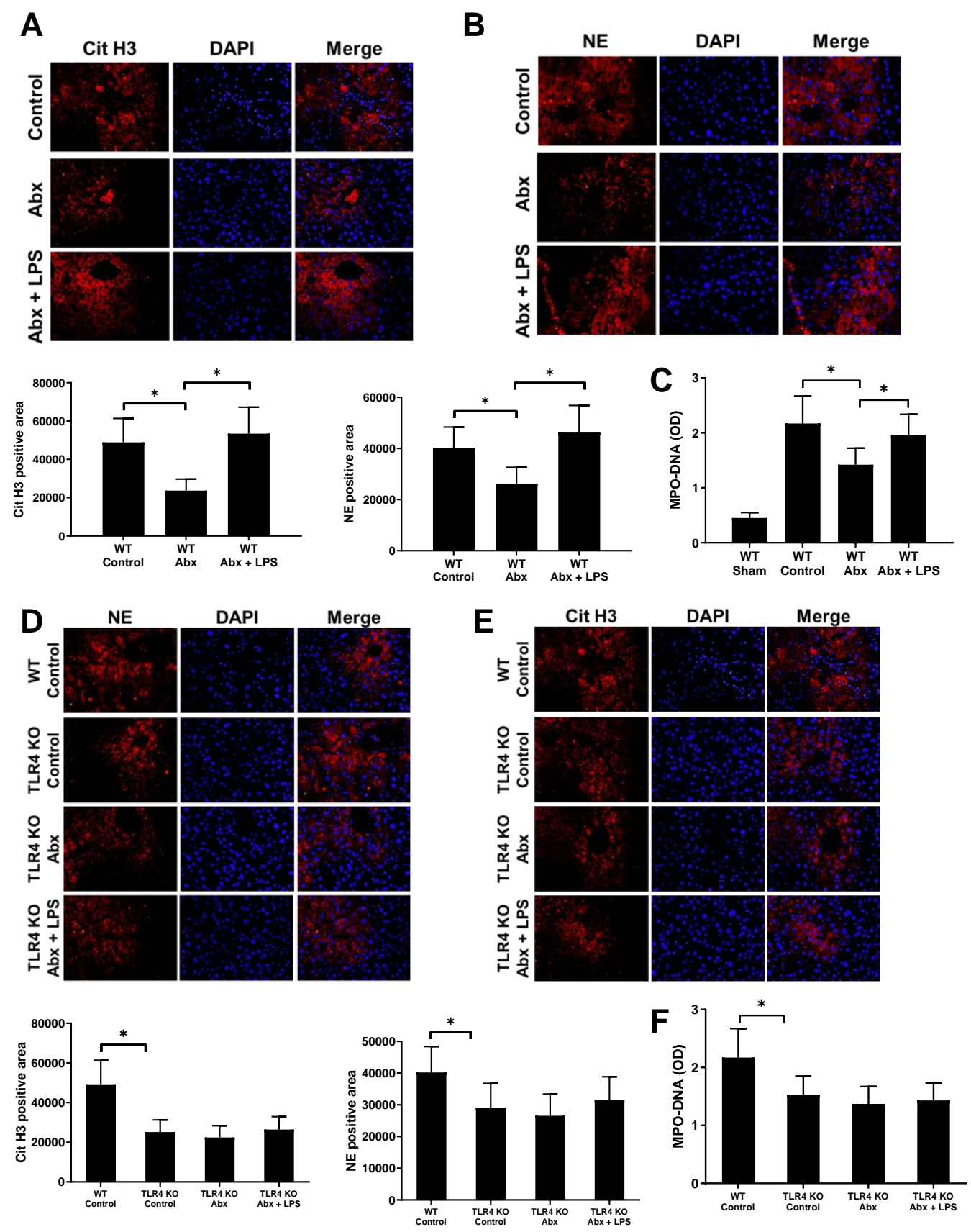
Figure 2
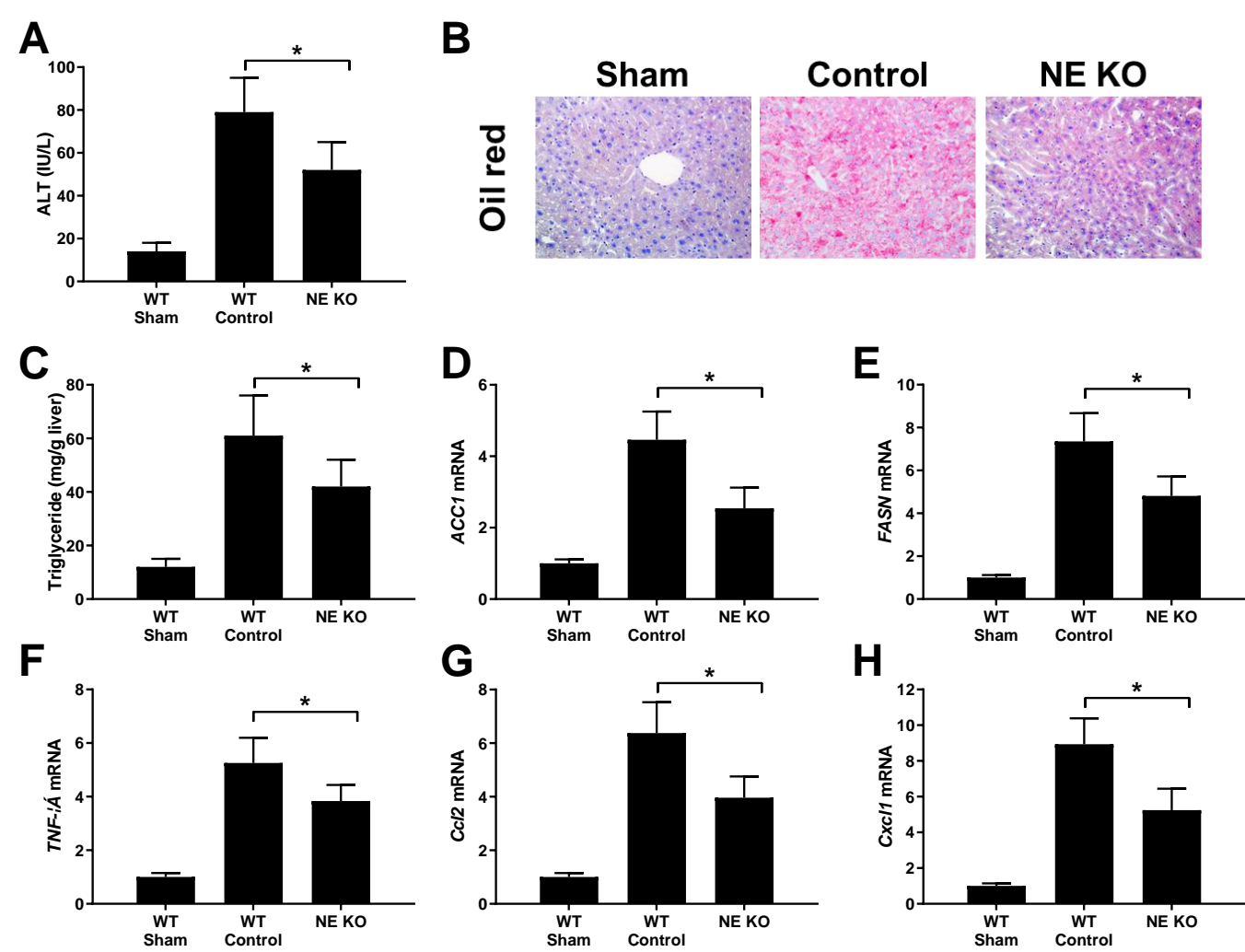

H
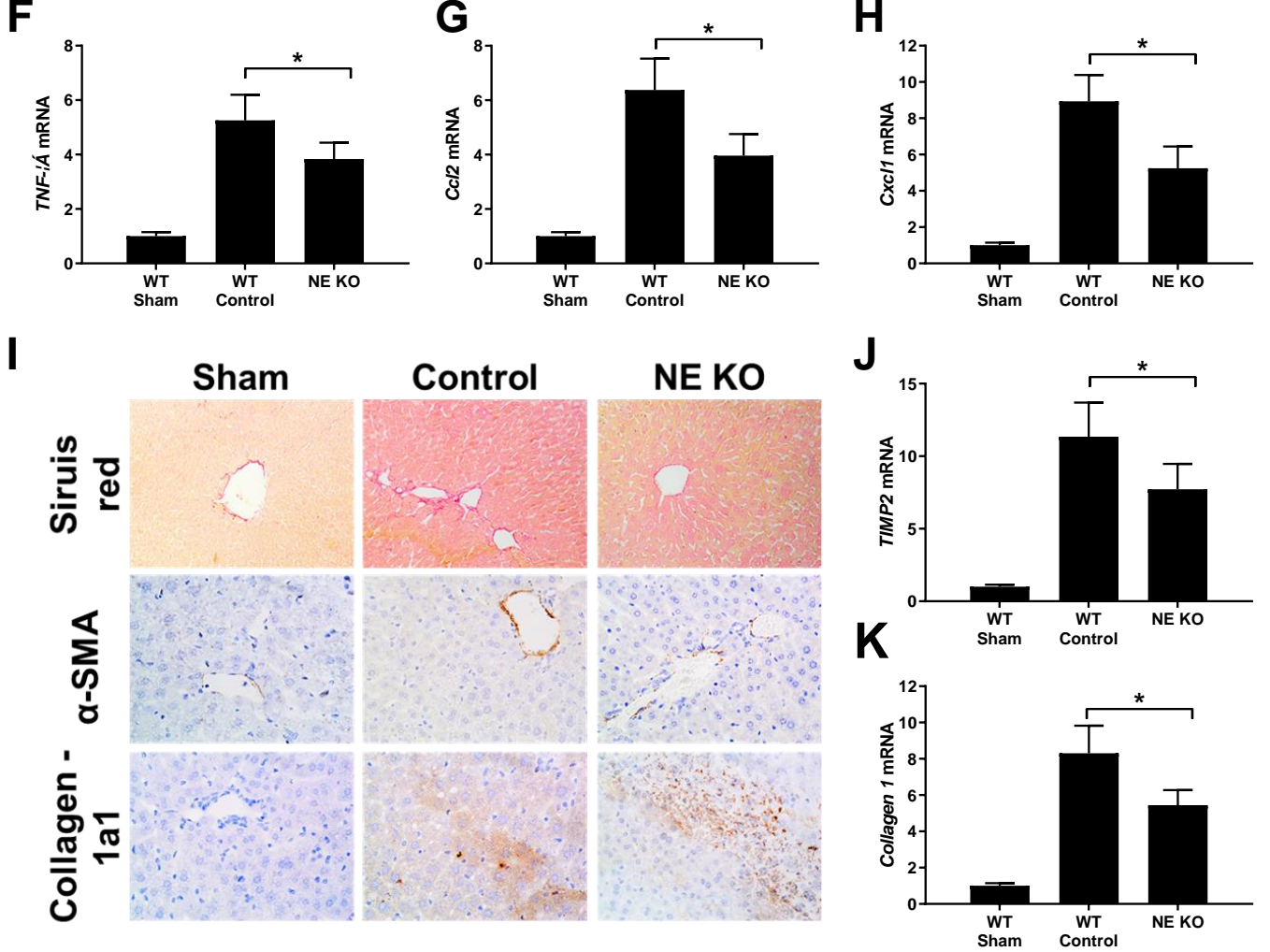
Figure 3
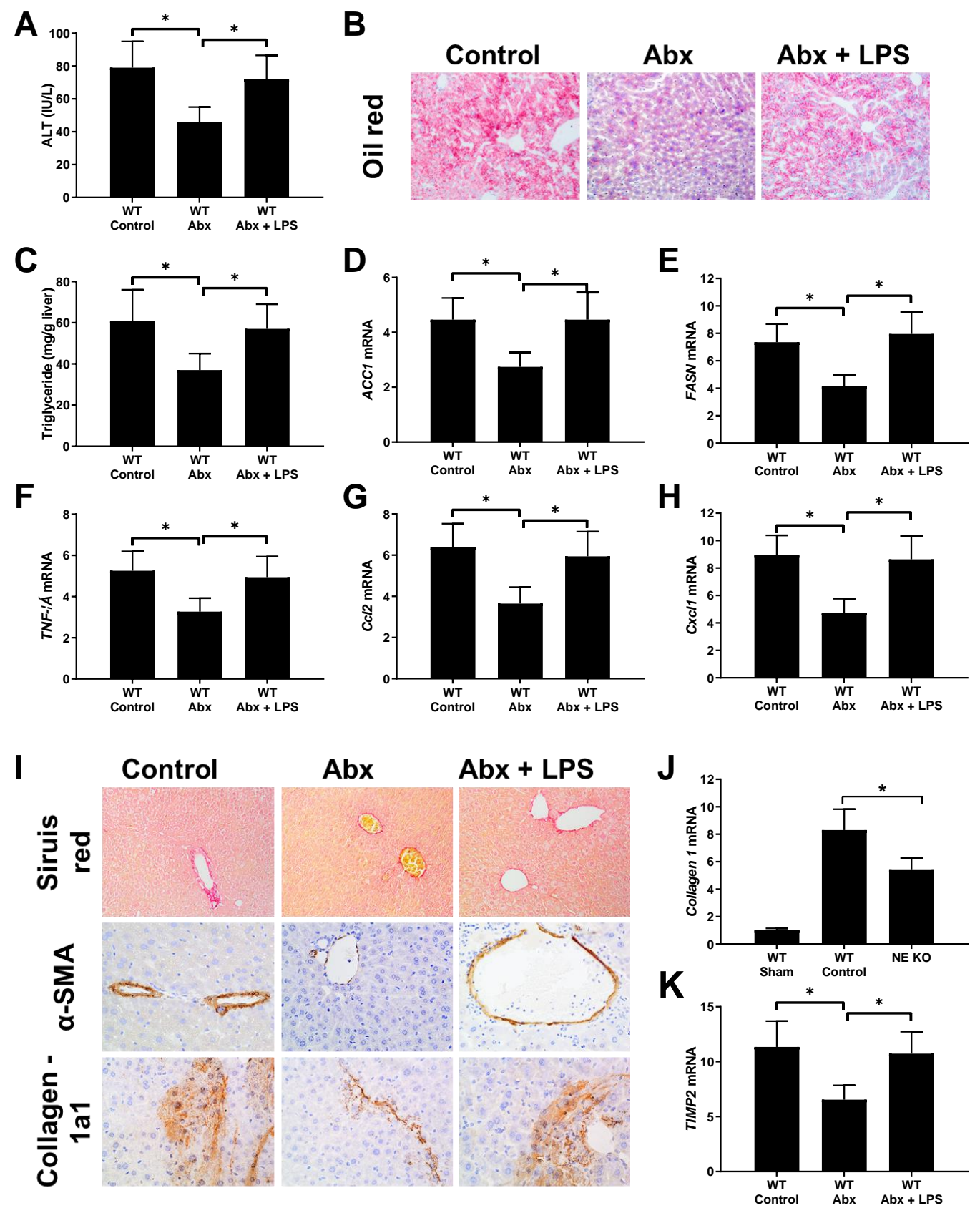
Figure 4

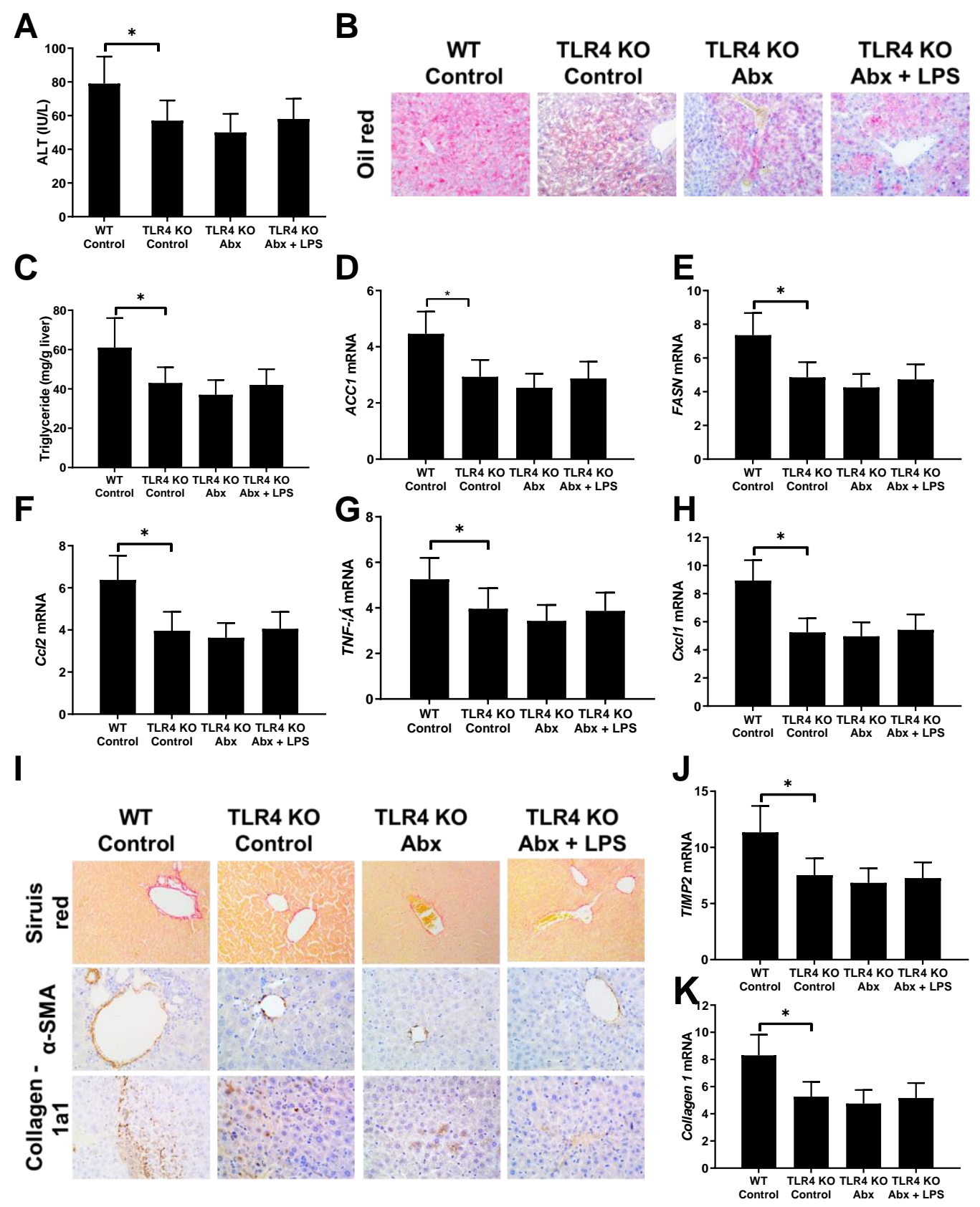

